\title{
QUASIHYPERBOLIC MAPPINGS IN BANACH SPACES
}

\author{
Qingshan Zhou \\ Foshan University, School of Mathematics and Big Data \\ Foshan, Guangdong 528000, P. R. China; q476308142@qq.com
}

\begin{abstract}
In this paper, we establish several characterizations for quasihyperbolic mappings in Banach spaces. As an application, we provide a partial solution to a problem left open by Väisälä regarding the local properties of quasihyperbolic mappings.
\end{abstract}

\section{Introduction and main results}

The quasihyperbolic metric (briefly, QH metric) was introduced by Gehring and his students Palka and Osgood in the 1970's in the setting of Euclidean spaces $\mathbf{R}^{n}$ $(n \geq 2)[5,6]$. Since its first appearance, the quasihyperbolic metric has become an important tool in geometric function theory of Euclidean spaces.

With the aid of the quasihyperbolic metric, from late 1980's onwards, Väisälä developed the theory of (dimension) free quasiconformal mappings in Banach spaces $[12,13,14,15,16]$. The main advantage of this approach avoids to make use of volume integrals and conformal modulus, which allows one to study the quasiconformality of mappings in Banach spaces with dimension infinity and metric spaces without volume measures. This research has recently attracted substantial interest in the research community (see e.g., [3, 7, 8, 10, 17] and reference therein).

In this paper, we will investigate a problem raised by Väisälä concerning the quasihyperbolic mappings in the free quasiconformal mapping theory ([16]). We first recall the definition of the quasihyperbolic mappings. In the following, we always assume that $E$ and $E^{\prime}$ are real Banach spaces with dimension at least 2 .

Definition 1.1. Let $D \subsetneq E$ and $D^{\prime} \subsetneq E^{\prime}$ be domains (open and connected), and let $f: D \rightarrow D^{\prime}$ be a homeomorphism. We say that $f$ is $M$-quasihyperbolic (or briefly $M-Q H)$ if for all $x$ and $y$ in $D$,

$$
\frac{1}{M} k_{D}(x, y) \leq k_{D^{\prime}}(f(x), f(y)) \leq M k_{D}(x, y),
$$

where $k_{D}$ and $k_{D^{\prime}}$ are the quasihyperbolic metrics of $D$ and $D^{\prime}$, respectively.

In geometric function theory, the class of quasihyperbolic mappings is closely related to the classes of conformal and quasiconformal mappings. It turns out that the quasihyperbolic mappings are useful in the study of quasiconformal extension of $\mathbf{R}^{n}$. For instance, Beurling and Ahlfors constructed a quasisymmetric extension from $\mathbf{R}^{1}$ to the half plane, which is actually quasihyperbolic [2]. In $\mathbf{R}^{n}$, when $n \neq 4$, Tukia and Väisälä demonstrated that two domains of $\mathbf{R}^{n}$ are quasihyperbolically equivalent if and only if they are quasiconformally equivalent [11].

In $[12,16]$, Väisälä systematically studied the properties of $\mathrm{QH}$ mappings and obtained the following results.

https://doi.org/10.5186/aasfm.2021.4619

2020 Mathematics Subject Classification: Primary 30C65, 30F45; Secondary 30C20.

Key words: Quasihyperbolic metric, quasihyperbolic mapping, quasisymmetric mapping, quasisimilarity. 
Theorem A. [12, Theorem 4.7] Suppose that $D \subsetneq E$ and $D^{\prime} \subsetneq E^{\prime}$ are domains, and that $f: D \rightarrow D^{\prime}$ is $M-Q H$. Then for any subdomain $G \subset D$, the restriction of $f$ on $G,\left.f\right|_{G}$, is $4 M^{2}-Q H$.

Theorem B. [16, Theorem 5.16] Suppose that $f: D \rightarrow D^{\prime}$ is a homeomorphism and that each point has a neighborhood $D_{1} \subset D$ such that $\left.f\right|_{D_{1}}: D_{1} \rightarrow f\left(D_{1}\right)$ is $M$-bilipschitz (or briefly $f$ is locally $M$-bilipschitz). Then $f$ is $M^{2}$-QH.

Obviously, not every QH mapping is bilipchitz (cf. [12, Section 4] or [16, Section 8]). Thus the following problem posed by Väisälä is natural.

Open Problem 1.1. [16, 13.2.13] Suppose that $f: D \rightarrow D^{\prime}$ is a homeomorphism and that each point has a neighborhood $D_{1} \subset D$ such that $\left.f\right|_{D_{1}}: D_{1} \rightarrow f\left(D_{1}\right)$ is $M$-QH. Is $f M^{\prime}$-QH with $M^{\prime}=M^{\prime}(M)$, where $M^{\prime}(M)$ means that the constant $M^{\prime}$ depends only on $M$ ?

The main motivation of this paper is to study Problem 1.1. As the first main result of this paper, we obtain the following equivalent characterizations of the quasihyperbolic mappings.

Theorem 1.1. Let $D \subsetneq E$ and $D^{\prime} \subsetneq E^{\prime}$ be domains, and let $f: D \rightarrow D^{\prime}$ be a homeomorphism. Then the following conditions are equivalent with the constants depending only on each other.

(1) $f: D \rightarrow D^{\prime}$ is $M-Q H$;

(2) $f$ and $f^{-1}$ are both $(L, \vartheta)$-locally bilipschitz;

(3) There are constants $C>0$ and $\mu \in(0,1)$ such that for any $x, y \in D$ with $|x-y|<\mu d_{D}(x)$

$$
\frac{1}{C} \frac{|x-y|}{d_{D}(x)} \leq \frac{|f(x)-f(y)|}{d_{D^{\prime}}(f(x))} \leq C \frac{|x-y|}{d_{D}(x)}
$$

(4) $f$ and $f^{-1}$ are both $\left(\theta, t_{0}\right)$-relative with $\theta(t)=M_{0} t$, where $0<t_{0}<1$ and $0<t<t_{0}$

(5) For all $x \in D, f: \mathbf{B}\left(x, d_{D}(x)\right) \rightarrow f\left(\mathbf{B}\left(x, d_{D}(x)\right)\right)$ is $M_{1}-Q H$ and $d_{D^{\prime}}(f(x)) \leq$ $M_{2} d_{f\left(\mathbf{B}\left(x, d_{D}(x)\right)\right)}(f(x))$.

Let us remark that the class of $(L, \vartheta)$-locally bilipschitz mappings in Theorem 1.1(2) (see Definition 2.2 below) was introduced by Bonk, Heinonen and Koskela when they established the theory of uniformizing Gromov hyperbolic spaces [3]. They also proved that a quasisymmetric and $(L, \vartheta)$-locally bilipschitz mapping between uniform metric spaces is quasihyperbolic (see [3, Proposition 4.36]). Based on Theorem 1.1, we shall generalize Proposition 4.36 in [3] to the setting of Banach spaces (see Lemma 3.5 below).

Also, we remark that the condition (1.2) is new and it is useful in the study of the local properties of the quasihyperbolic mappings. Note that the $\theta$-relative mappings stated in Theorem 1.1(4) were first considered by Gehring, and also, the relationship between $\theta$-relative mappings and quasiconformal mappings in $\mathbf{R}^{n}$ was investigated [4].

With the aid of Theorem 1.1, we obtain the following partial solution to Problem 1.1.

Theorem 1.2. Suppose that $f: D \rightarrow D^{\prime}$ is a homeomorphism. If there exist a constant $M>1$ and a homeomorphism $\eta:[0, \infty) \rightarrow[0, \infty)$ such that for each point $x \in D, f: \mathbf{B}\left(x, d_{D}(x)\right) \rightarrow f\left(\mathbf{B}\left(x, d_{D}(x)\right)\right)$ is $M$-quasihyperbolic and $\eta$ quasisymmetric, then $f$ is $M_{1}-Q H$ with $M_{1}=M_{1}(M, \eta)$. 
We remark that the authors in [7] recently considered Problem 1.1 in metric spaces. However, the target spaces in [7] are always assumed to be proper. Here a metric space being proper means that every closed ball in this space is compact. Obviously, in all Banach spaces with dimension infinity, this condition is invalid, and also, this condition plays a key role in the discussions in [7]. This shows that the proof methods in [7] cannot be used to prove Theorem 1.2 in the setting of Banach spaces with dimension infinity. We shall establish a different method to overcome this difficulty.

Further, by Theorems 1.1 and 1.2, we get the following result.

Theorem 1.3. Suppose that $f: D \rightarrow D^{\prime}$ is a homeomorphism such that for each point $x \in D,\left.f\right|_{\mathbf{B}\left(x, d_{D}(x)\right)}$ is quasisimilar with data $(\eta, L, \lambda)$. Then $f$ is $M-Q H$ with $M=M(\eta, L, \lambda)$.

Remark 1.1. The concept of quasisimilarity was introduced in [3] (see Definition 2.4 below). In [3], the authors demonstrated that there is a one-to-one correspondence between the quasi-isometry classes of Gromov hyperbolic spaces and the quasisimilarity classes of uniform metric spaces (see [3, Theorem 1.1]). Note that, in $[1,9]$, the authors also considered quasisimilar mappings in infinite-dimensional Banach spaces. But these two classes of mappings are not the same.

The rest of this paper is organized as follows. In Section 2, we recall necessary definitions and preliminary results, and the proofs of Theorems $1.1 \sim 1.3$ are presented in Section 3.

\section{Preliminaries}

In this section, we recall some necessary terminology. Throughout this paper, we always assume that $E$ and $E^{\prime}$ denote real Banach spaces with dimension at least 2, and that $D \subsetneq E$ and $D^{\prime} \subsetneq E^{\prime}$ are domains. The norm of a vector $z$ in $E$ is written as $|z|$, and for each pair of points $z_{1}, z_{2}$ in $E$, the distance between them is denoted by $\left|z_{1}-z_{2}\right|$, the closed line segment with endpoints $z_{1}$ and $z_{2}$ by $\left[z_{1}, z_{2}\right]$. The distance from $z \in D$ to the boundary $\partial D$ of $D$ is denoted by $d_{D}(z)$. For a bounded set $A$ in $E, \operatorname{diam} A$ means the diameter of $A$.

Let

$$
\mathbf{B}(x, r)=\{z \in E:|z-x|<r\}, \quad \overline{\mathbf{B}}(x, r)=\{z \in E:|z-x| \leq r\},
$$

and

$$
\mathbf{S}(x, r)=\{z \in E:|z-x|=r\} .
$$

The quasihyperbolic length of a rectifiable arc or a curve $\alpha$ with respect to the norm metric in a domain $D \subsetneq E$ is the number (cf. $[5,6]$ ):

$$
\ell_{k}(\alpha)=\int_{\alpha} \frac{|d z|}{d_{D}(z)}
$$

For each pair of points $z_{1}, z_{2}$ in $D$, the quasihyperbolic distance $k_{D}\left(z_{1}, z_{2}\right)$ between $z_{1}$ and $z_{2}$ is defined in the usual way:

$$
k_{D}\left(z_{1}, z_{2}\right)=\inf \ell_{k}(\alpha),
$$

where the infimum is taken over all rectifiable curves $\alpha$ joining $z_{1}$ to $z_{2}$ in $D$.

For all $z_{1}, z_{2}$ in $D$, we have the following chain of inequalities (cf. [16])

$$
k_{D}\left(z_{1}, z_{2}\right) \geq \log \left(1+\frac{\left|z_{1}-z_{2}\right|}{\min \left\{d_{D}\left(z_{1}\right), d_{D}\left(z_{2}\right)\right\}}\right) \geq\left|\log \frac{d_{D}\left(z_{2}\right)}{d_{D}\left(z_{1}\right)}\right| .
$$


Moreover, we record the following useful result.

Theorem C. [16, Theorem 3.9] Let $D \subsetneq E$ be a domain, and let $x, y \in D$ be such that $|x-y| \leq \frac{1}{2} d_{D}(x)$ or $k_{D}(x, y) \leq 1$. Then

$$
\frac{1}{2} \frac{|x-y|}{d_{D}(x)}<k_{D}(x, y) \leq 2 \frac{|x-y|}{d_{D}(x)} .
$$

Next, we recall several concepts from $[3,16]$.

Definition 2.1. Let $0<t_{0} \leq 1$, and let $\theta:\left[0, t_{0}\right) \rightarrow[0, \infty)$ be an embedding with $\theta(0)=0$. Suppose that $f: D \rightarrow D^{\prime}$ is a homeomorphism with $D \neq E$ and $D^{\prime} \neq E^{\prime}$. We say that $f$ is $\left(\theta, t_{0}\right)$-relative if

$$
\frac{|f(x)-f(y)|}{d_{D^{\prime}}(f(x))} \leq \theta\left(\frac{|x-y|}{d_{D}(x)}\right)
$$

for all $x, y \in D$ with $|x-y|<t_{0} d_{D}(x)$. If $t_{0}=1$, then we say that $f$ is $\theta$-relative.

Definition 2.2. Let $D \subsetneq E$ and $D^{\prime} \subsetneq E^{\prime}$ be domains. A homeomorphism $f: D \rightarrow D^{\prime}$ is said to be $(L, \lambda)$-locally bilipschitz, where $L>0$ and $\lambda \in(0,1)$, if for each $x \in D$, there is a constant $c_{x}>0$ such that for all $y, z \in B\left(x, \lambda d_{D}(x)\right)$,

$$
\frac{c_{x}}{L}|y-z| \leq|f(y)-f(z)| \leq L c_{x}|y-z| .
$$

Definition 2.3. A homeomorphism $f: X \rightarrow X^{\prime}$ between two metric spaces is said to be $\eta$-quasisymmetric if there is a homeomorphism $\eta:[0, \infty) \rightarrow[0, \infty)$ such that

$$
d(x, a) \leq t d(x, b) \quad \text { implies } \quad d^{\prime}(f(x), f(a)) \leq \eta(t) d^{\prime}(f(x), f(b))
$$

for each $t>0$ and for each triplet $x, a, b$ of points in $X$.

Definition 2.4. Let $D \subsetneq E$ and $D^{\prime} \subsetneq E^{\prime}$ be domains. A homeomorphism $f: D \rightarrow D^{\prime}$ is said to be quasisimilar with data $(\eta, L, \lambda)$ if it is both $\eta$-quasisymmetric and $(L, \lambda)$-locally bilipschitz.

\section{Proofs of main results}

3.1. Proof of Theorem 1.1. The proof consists of four lemmas: Lemma 3.1 $\sim$ Lemma 3.4. Precisely, the equivalent relationships $(1) \Longleftrightarrow(2) \Longleftrightarrow(3)$ are proved in Lemma 3.1; The implications $(1) \Rightarrow(4) \Rightarrow(3)$ are demonstrated in Lemmas 3.2 and 3.3 , and the equivalent relationship $(1) \Longleftrightarrow(5)$ is shown in Lemma 3.4.

Lemma 3.1. Let $D \subsetneq E$ and $D^{\prime} \subsetneq E^{\prime}$ be domains, and let $f: D \rightarrow D^{\prime}$ be a homeomorphism. Then the following conditions are equivalent with constants depending only on each other:

(1) $f: D \rightarrow D^{\prime}$ is $M-Q H$;

(2) $f$ and $f^{-1}$ are both $(L, \vartheta)$-locally bilipschitz;

(3) there are constants $C>0$ and $\mu \in(0,1)$ such that for all $x, y \in D$ with $|x-y|<\mu d_{D}(x)$,

$$
\frac{1}{C} \frac{|x-y|}{d_{D}(x)} \leq \frac{|f(x)-f(y)|}{d_{D^{\prime}}(f(x))} \leq C \frac{|x-y|}{d_{D}(x)} .
$$

Proof. We prove this lemma by showing the implications $(1) \Rightarrow(2) \Rightarrow(3) \Rightarrow(1)$.

$(1) \Rightarrow(2)$ : Assume that $f$ is $M$-QH. Obviously, we only need to show that $f$ is $(L, \vartheta)$-locally bilipschitz for some suitable constants $L \geq 1$ and $\vartheta \in(0,1)$ both depending only on $M$. 
For any $z \in D$ and for any $x, y \in \mathbf{B}\left(z, \frac{1}{4 M+1} d_{D}(z)\right)$, we have

which implies

$$
|x-y| \leq \frac{2}{4 M+1} d_{D}(z) \quad \text { and } \quad d_{D}(x) \geq \frac{4 M}{4 M+1} d_{D}(z),
$$

Then, by Lemma C, we have

$$
|x-y| \leq \frac{1}{2 M} d_{D}(x)
$$

$$
k_{D}(x, y) \leq 2 \frac{|x-y|}{d_{D}(x)} \leq \frac{1}{M}
$$

Moreover, since $f$ is $M$-QH, we get from (2.1) that

$$
\begin{aligned}
\log \left(1+\frac{|f(x)-f(y)|}{d_{D^{\prime}}(f(x))}\right) & \leq k_{D^{\prime}}(f(x), f(y)) \leq M k_{D}(x, y) \\
& \leq \min \left\{2 M \frac{|x-y|}{d_{D}(x)}, 1\right\}
\end{aligned}
$$

and thus,

$$
|f(x)-f(y)| \leq(e-1) d_{D^{\prime}}(f(x)) .
$$

This yields

$$
\frac{|f(x)-f(y)|}{d_{D^{\prime}}(f(x))} \leq e \log \left(1+\frac{|f(x)-f(y)|}{d_{D^{\prime}}(f(x))}\right) \leq 2 M e \frac{|x-y|}{d_{D}(x)} .
$$

On the other hand, by (3.2), we see that

$$
k_{D^{\prime}}(f(x), f(y)) \leq 1 .
$$

Again, by Lemma C, together with (2.1), we know that

$$
\log \left(1+\frac{|x-y|}{d_{D}(x)}\right) \leq k_{D}(x, y) \leq M k_{D^{\prime}}(f(x), f(y)) \leq \min \left\{2 M \frac{|f(x)-f(y)|}{d_{D^{\prime}}(f(x))}, M\right\} .
$$

This leads to

$$
|x-y| \leq\left(e^{M}-1\right) d_{D}(x)
$$

and so,

$$
\frac{|x-y|}{d_{D}(x)} \leq e^{M} \log \left(1+\frac{|x-y|}{d_{D}(x)}\right) \leq 2 M e^{M} \frac{|f(x)-f(y)|}{d_{D^{\prime}}(f(x))} .
$$

By letting $L=2 M e^{M}$ and $\vartheta=\frac{1}{4 M+1}$, we know that $f$ is $(L, \vartheta)$-locally bilipschitz.

$(2) \Rightarrow(3)$ : Assume that both $f$ and $f^{-1}$ are $(L, \vartheta)$-locally bilipschitz for constants $L \geq 1$ and $\vartheta \in(0,1)$. Let $\mu=\frac{\vartheta^{2}}{4 L^{2}}$. We are going to prove that there exists some constant $C \geq 1$ such that for any $x, y \in D$ with $|x-y|<\mu d_{D}(x)$, (3.1) holds.

We first check the right hand side of the inequalities (3.1). Since $f$ is $(L, \vartheta)$-locally bilipschitz, we see that there is a constant $c_{x}>0$ such that for all $u \in \mathbf{B}\left(x, \vartheta d_{D}(x)\right)$,

$$
\frac{c_{x}}{L}|x-u| \leq|f(x)-f(u)| \leq L c_{x}|x-u| .
$$

Let $a^{\prime} \in \partial D^{\prime}$ be such that

$$
\left|f(x)-a^{\prime}\right| \leq 2 d_{D^{\prime}}(f(x))
$$

and let $w$ be such that

$$
|x-w|=\frac{1}{2} \vartheta d_{D}(x) \quad \text { and } \quad w^{\prime}=f(w) \in\left[f(x), a^{\prime}\right) \cap f\left(\mathbf{B}\left(x, \vartheta d_{D}(x)\right)\right) .
$$


Then it follows from (3.3) that

$$
\frac{\vartheta c_{x}}{2 L} d_{D}(x)=\frac{c_{x}}{L}|w-x| \leq\left|w^{\prime}-f(x)\right| \leq\left|a^{\prime}-f(x)\right| \leq 2 d_{D^{\prime}}(f(x)),
$$

and so,

$$
|f(x)-f(y)| \leq L c_{x}|x-y| \leq \frac{4 L^{2}}{\vartheta} \frac{|x-y|}{d_{D}(x)} d_{D^{\prime}}(f(x)) .
$$

Since (3.4) and the choice of $\mu$ guarantee that

$$
|f(x)-f(y)| \leq \vartheta d_{D^{\prime}}(f(x)),
$$

and since $f^{-1}$ is $(L, \vartheta)$-locally bilipschitz, similarly, we know that the left hand side of the inequalities (3.1) is also true.

$(3) \Rightarrow(1)$ : Assume that there are constants $C>0$ and $\mu \in(0,1)$ such that for all $x, y \in D$ with $|x-y|<\mu d_{D}(x)$, (3.1) holds true. Then we infer from (3.1) that

$$
L(x, f):=\limsup _{y \rightarrow x} \frac{|f(y)-f(x)|}{|y-x|} \leq \frac{C d_{D^{\prime}}(f(x))}{d_{D}(x)}
$$

and

$$
l(x, f):=\liminf _{y \rightarrow x} \frac{|f(y)-f(x)|}{|y-x|} \geq \frac{d_{D^{\prime}}(f(x))}{C d_{D}(x)} .
$$

Thus [16, Theorem 5.6] ensures that $f$ is $C-\mathrm{QH}$.

Lemma 3.2. Let $D \subsetneq E$ and $D^{\prime} \subsetneq E^{\prime}$ be domains, and let $f: D \rightarrow D^{\prime}$ be a homeomorphism. If both $f$ and $f^{-1}$ are $\left(\theta, t_{0}\right)$-relative with $\theta(t)=M_{0} t$, where $0<t_{0}<1$ and $0<t<t_{0}$, then there are constants $C>0$ and $\mu \in(0,1)$ such that for all $x, y \in D$ with $|x-y|<\mu d_{D}(x)$,

$$
\frac{1}{C} \frac{|x-y|}{d_{D}(x)} \leq \frac{|f(x)-f(y)|}{d_{D^{\prime}}(f(x))} \leq C \frac{|x-y|}{d_{D}(x)} .
$$

Proof. Let $\mu=\frac{t_{0}}{M_{0}}$. Since $f$ is $\left(\theta, t_{0}\right)$-relative, it follows that for all $x, y \in D$ with $|x-y|<\mu d_{D}(x)$,

$$
\frac{|f(x)-f(y)|}{d_{D^{\prime}}(f(x))} \leq M_{0} \frac{|x-y|}{d_{D}(x)}
$$

from which the right hand side of the inequalities (3.5) follows.

Because (3.6) and the choice of $\mu$ guarantee that

$$
|f(x)-f(y)|<t_{0} d_{D^{\prime}}(f(x)),
$$

and since $f^{-1}$ is $\left(\theta, t_{0}\right)$-relative, we deduce that

$$
\frac{|x-y|}{d_{D}(x)} \leq M_{0} \frac{|f(x)-f(y)|}{d_{D^{\prime}}(f(x))} .
$$

Hence the lemma is proved.

Lemma 3.3. Let $D \subsetneq E$ and $D^{\prime} \subsetneq E^{\prime}$ be domains, and let $f: D \rightarrow D^{\prime}$ be a homeomorphism. If $f$ is $M-Q H$, then both $f$ and $f^{-1}$ are $\left(\theta, t_{0}\right)$-relative with $\theta(t)=M_{0} t$, where $0<t_{0}<1$ and $0<t<t_{0}$. 
Proof. For any $x, y \in D$, if $|x-y|<\frac{1}{2 M} d_{D}(x)$, then we see from Lemma C that

$$
k_{D^{\prime}}(f(x), f(y)) \leq M k_{D}(x, y) \leq 2 M \frac{|x-y|}{d_{D}(x)} \leq 1
$$

and so,

$$
\frac{1}{2} \frac{|f(x)-f(y)|}{d_{D^{\prime}}(f(x))} \leq k_{D^{\prime}}(f(x), f(y)) \leq 2 M \frac{|x-y|}{d_{D}(x)} .
$$
$M_{0} t$.

By letting $t_{0}=\frac{1}{2 M}$ and $M_{0}=4 M$, we know that $f$ is $\left(\theta, t_{0}\right)$-relative with $\theta(t)=$

Similarly, we see that $f^{-1}$ is also $\left(\theta, t_{0}\right)$-relative. Hence the lemma is proved.

Lemma 3.4. Let $D \subsetneq E$ and $D^{\prime} \subsetneq E^{\prime}$ be domains, and let $f: D \rightarrow D^{\prime}$ be a homeomorphism. Then the following conditions are equivalent:

(1) $f: D \rightarrow D^{\prime}$ is $M-Q H$;

(2) For any $x \in D$, the restriction $f: \mathbf{B}\left(x, d_{D}(x)\right) \rightarrow f\left(\mathbf{B}\left(x, d_{D}(x)\right)\right)$ is $M_{1}-Q H$ and

$$
d_{D^{\prime}}(f(x)) \leq M_{2} d_{f\left(\mathbf{B}\left(x, d_{D}(x)\right)\right)}(f(x)) .
$$

Proof. $(1) \Rightarrow(2)$ : Assume that $f: D \rightarrow D^{\prime}$ is $M$-QH. It follows from Theorem A that for any $x \in D$, the restriction $f: \mathbf{B}\left(x, d_{D}(x)\right) \rightarrow f\left(\mathbf{B}\left(x, d_{D}(x)\right)\right)$ is $M_{1}$-QH with $M_{1}=4 M^{2}$.

Fix $x \in D$. On the one hand, since $f: D \rightarrow D^{\prime}$ is $M$-QH, we deduce from Lemma 3.1 that there are constants $C=C(M) \geq 1$ and $\mu=\mu(M) \in(0,1)$ such that for all $x, y \in D$ with $|x-y|<\mu d_{D}(x)$,

$$
\frac{1}{C} \frac{|x-y|}{d_{D}(x)} \leq \frac{|f(x)-f(y)|}{d_{D^{\prime}}(f(x))} \leq C \frac{|x-y|}{d_{D}(x)}
$$

On the other hand, since $f: \mathbf{B}\left(x, d_{D}(x)\right) \rightarrow f\left(\mathbf{B}\left(x, d_{D}(x)\right)\right)$ is $M_{1}$-QH, again, by Lemma 3.1, it follows that there are constants $C_{1}=C_{1}(M) \geq 1$ and $\mu_{1}=\mu_{1}(M) \in$ $(0,1)$ such that for all $x, y \in D$ with $|x-y|<\mu_{1} d_{D}(x)$,

$$
\frac{1}{C_{1}} \frac{|x-y|}{d_{\mathbf{B}\left(x, d_{D}(x)\right)}(x)} \leq \frac{|f(x)-f(y)|}{d_{f\left(\mathbf{B}\left(x, d_{D}(x)\right)\right)}(f(x))} \leq C_{1} \frac{|x-y|}{d_{\mathbf{B}\left(x, d_{D}(x)\right)}(x)} .
$$

Note that $d_{\mathbf{B}\left(x, d_{D}(x)\right)}(x)=d_{D}(x)$. By taking $M_{2}=C C_{1}$, we know that

$$
d_{D^{\prime}}(f(x)) \leq M_{2} d_{f\left(\mathbf{B}\left(x, d_{D}(x)\right)\right)}(f(x)) .
$$

$(2) \Rightarrow(1)$ : Because $f: \mathbf{B}\left(x, d_{D}(x)\right) \rightarrow f\left(\mathbf{B}\left(x, d_{D}(x)\right)\right)$ is $M_{1}$-QH, it follows from Lemma 3.1 that there exist constants $C_{2}=C_{2}\left(M_{1}\right) \geq 1$ and $\mu_{2}=\mu_{2}\left(M_{1}\right) \in(0,1)$ such that for all $x, y \in D$ with $|x-y|<\mu_{2} d_{\mathbf{B}\left(x, d_{D}(x)\right)}(x)$,

$$
\frac{1}{C_{2}} \frac{|x-y|}{d_{\mathbf{B}\left(x, d_{D}(x)\right)}(x)} \leq \frac{|f(x)-f(y)|}{d_{f\left(\mathbf{B}\left(x, d_{D}(x)\right)\right)}(f(x))} \leq C_{2} \frac{|x-y|}{d_{\mathbf{B}\left(x, d_{D}(x)\right)}(x)} .
$$

Then the facts

$$
d_{f\left(\mathbf{B}\left(x, d_{D}(x)\right)\right)}(f(x)) \leq d_{D^{\prime}}(f(x)) \leq M_{2} d_{f\left(\mathbf{B}\left(x, d_{D}(x)\right)\right)}(f(x))
$$

lead to

$$
\frac{1}{C_{2} M_{2}} \frac{|x-y|}{d_{D}(x)} \leq \frac{|f(x)-f(y)|}{d_{D^{\prime}}(f(x))} \leq C_{2} \frac{|x-y|}{d_{D}(x)} .
$$

Once more, by Lemma 3.1, we know that $f: D \rightarrow D^{\prime}$ is $M$-QH with constant $M=$ $M\left(\mu_{2}, C_{2}, M_{2}\right)=M\left(M_{1}, M_{2}\right)$. 
3.2. Proof of Theorem 1.2. Denote by $B_{x}=\mathbf{B}\left(x, d_{D}(x)\right)$ for $x \in D$. By [15, Theorem 6.12], we may assume that $f$ is $\eta$-quasisymmetric on all closed balls $\overline{\mathbf{B}}\left(x, d_{D}(x)\right)$ for $x \in D$. To prove that $f$ is $M$-QH, by Theorem 1.1 , we only need to check that for any $x_{0} \in D$,

$$
d_{D^{\prime}}\left(f\left(x_{0}\right)\right) \leq 2 \eta(1) d_{f\left(B_{x_{0}}\right)}\left(f\left(x_{0}\right)\right) .
$$

Let $\varepsilon \in(0,1 / 100)$ be such that

$$
\eta(1) \eta(\varepsilon) \leq \frac{1}{2}
$$

First, the similar reasoning as in the proof of [13, Theorem 3.10] guarantees that there is a sequence of points $\left\{x_{i}\right\}_{i \in \mathbf{N}}$ and an end cut $\gamma$ of $D$ emanating from $x_{0}$ such that

(1) $\gamma_{n}=\cup_{i=0}^{n-1}\left[x_{i}, x_{i+1}\right)$ and $\gamma=\cup_{n=0}^{\infty} \gamma_{n}$, where $\left[x_{i}, x_{i+1}\right]$ is a line segment in $\bar{D}$;

(2) $x_{i+1} \in \mathbf{S}\left(x_{i}, d_{D}\left(x_{i}\right)\right)$ and $d_{D}\left(x_{i+1}\right) \leq \varepsilon_{i+1} d_{D}\left(x_{i}\right)$, and

(3) $\lim _{n \rightarrow \infty} x_{n}=a \in \partial D$.

No loss of generality, we assume that $x_{i} \in D$ for all $i \in \mathbf{N}$. Then we claim that for all $i \in \mathbf{N}$,

$$
\left|f\left(x_{i+1}\right)-f\left(x_{i+2}\right)\right| \leq \frac{1}{2}\left|f\left(x_{i}\right)-f\left(x_{i+1}\right)\right| .
$$

To prove this claim, for each $i \in \mathbf{N}$, take a point

$$
z_{i+1} \in \mathbf{S}\left(x_{i+1}, d_{G}\left(x_{i+1}\right)\right) \cap \mathbf{S}\left(x_{i}, d_{G}\left(x_{i}\right)\right)
$$

with $z_{i+1} \neq x_{i+2}$. Then

$$
\frac{\left|f\left(x_{i+2}\right)-f\left(x_{i+1}\right)\right|}{\left|f\left(z_{i+1}\right)-f\left(x_{i+1}\right)\right|} \leq \eta\left(\frac{\left|x_{i+2}-x_{i+1}\right|}{\left|z_{i+1}-x_{i+1}\right|}\right)=\eta(1)
$$

since $f$ is $\eta$-quasisymmetric on $\overline{\mathbf{B}}\left(x_{i+1}, d_{D}\left(x_{i+1}\right)\right)$. Also, we have

$$
\frac{\left|f\left(x_{i+1}\right)-f\left(z_{i+1}\right)\right|}{\left|f\left(x_{i+1}\right)-f\left(x_{i}\right)\right|} \leq \eta\left(\frac{\left|x_{i+1}-z_{i+1}\right|}{\left|x_{i+1}-x_{i}\right|}\right) \leq \eta\left(\varepsilon_{i+1}\right),
$$

since $f$ is $\eta$-quasisymmetric on $\overline{\mathbf{B}}\left(x_{i}, d_{D}\left(x_{i}\right)\right)$ and $d_{D}\left(x_{i+1}\right) \leq \varepsilon_{i+1} d_{D}\left(x_{i}\right)$.

These ensure that

$$
\begin{aligned}
\left|f\left(x_{i+1}\right)-f\left(x_{i+2}\right)\right| & \leq \eta(1)\left|f\left(x_{i+1}\right)-f\left(z_{i+1}\right)\right| \\
& \leq \eta(1) \eta(\varepsilon)\left|f\left(x_{i}\right)-f\left(x_{i+1}\right)\right| \\
& \leq \frac{1}{2}\left|f\left(x_{i}\right)-f\left(x_{i+1}\right)\right|
\end{aligned}
$$

and so, the claim is true.

Now, it follows from the claim that

$$
\left|f\left(x_{0}\right)-f\left(x_{n+1}\right)\right| \leq \sum_{i=0}^{n}\left|f\left(x_{i}\right)-f\left(x_{i+1}\right)\right| \leq 2\left|f\left(x_{0}\right)-f\left(x_{1}\right)\right|,
$$

and thus, we infer from the condition $\lim _{n \rightarrow \infty} x_{n}=a \in \partial D$ that

$$
d_{D^{\prime}}\left(f\left(x_{0}\right)\right) \leq\left|f\left(x_{0}\right)-f(a)\right| \leq 2\left|f\left(x_{0}\right)-f\left(x_{1}\right)\right| .
$$

Moreover, we have that for any point $w \in \mathbf{S}\left(x_{0}, d_{D}\left(x_{0}\right)\right)$,

$$
\left|f\left(x_{0}\right)-f\left(x_{1}\right)\right| \leq \eta(1)\left|f\left(x_{0}\right)-f(w)\right|,
$$


since $f$ is $\eta$-quasisymmetric on the closed ball $\overline{\mathbf{B}}\left(x_{0}, d_{D}\left(x_{0}\right)\right)$. Hence

$$
\left|f\left(x_{0}\right)-f\left(x_{1}\right)\right| \leq \eta(1) d_{f\left(B_{x_{0}}\right)}\left(f\left(x_{0}\right)\right) .
$$

Easily, (3.7) follows from (3.8) and (3.9), and so, the proof of Theorem 1.2 is complete.

3.3. Proof of Theorem 1.3. We start the proof with the following auxiliary result.

Lemma 3.5. Let $D \subsetneq E$ and $D^{\prime} \subsetneq E^{\prime}$ be domains, and let $f: D \rightarrow D^{\prime}$ be a homeomorphism. If $f$ is $\eta$-quasisymmetric and $(L, \lambda)$-locally bilipschitz, then $f$ is $M-Q H$ with $M=M(\eta, L, \lambda)$.

Proof. To prove the theorem, by Theorem 1.1, we only need to show that $f^{-1}$ is $\left(L^{\prime}, \lambda^{\prime}\right)$-locally bilipschitz. Since $f$ is $(L, \lambda)$-locally bilipschitz, it is enough to check that there is a constant $\lambda^{\prime}=\lambda^{\prime}(\eta, \lambda)$ such that for $x^{\prime} \in D^{\prime}$,

$$
f^{-1}\left(\mathbf{B}\left(x^{\prime}, \lambda^{\prime} d_{D^{\prime}}\left(x^{\prime}\right)\right)\right) \subset \mathbf{B}\left(x, \lambda d_{D}(x)\right),
$$

where $x=f^{-1}\left(x^{\prime}\right)$.

To this end, let $\lambda^{\prime} \in\left(0, \frac{1}{2}\right)$ be such that

$$
\eta^{\prime}\left(\lambda^{\prime}\right)<\lambda
$$

where $\eta^{\prime}(t)=\eta^{-1}\left(t^{-1}\right)^{-1}$.

Fix $x^{\prime} \in D^{\prime}$, and let $x=f^{-1}\left(x^{\prime}\right)$. For any $y^{\prime} \in \mathbf{B}\left(x^{\prime}, \lambda^{\prime} d_{D^{\prime}}\left(x^{\prime}\right)\right)$, set $y=f^{-1}\left(y^{\prime}\right)$. Since $f$ is $\eta$-quasisymmetric, we know that the inverse map $f^{-1}: D^{\prime} \rightarrow D$ is $\eta^{\prime}$ quasisymmetric. Moreover, it follows from [15, Theorem 6.12] that $f^{-1}$ can be extended to an $\eta^{\prime}$-quasisymmetric mapping from $\overline{D^{\prime}}$ to $\bar{D}$, which is still denoted by $f^{-1}$ for simplicity.

For any $u \in \partial D$, we have

$$
\frac{|x-y|}{|x-u|} \leq \eta^{\prime}\left(\frac{|f(x)-f(y)|}{|f(x)-f(u)|}\right) \leq \eta^{\prime}\left(\lambda^{\prime}\right)<\lambda .
$$

Then the arbitrariness of $u \in \partial D$ implies

$$
|x-y|<\lambda d_{D}(x) .
$$

Therefore, the relation (3.10) holds true.

Now, we are ready to prove Theorem 1.3. Assume that for each $x \in D$, the restriction $\left.f\right|_{\mathbf{B}\left(x, d_{D}(x)\right)}$ is both $\eta$-quasisymmetric and $(L, \lambda)$-locally bilipschitz. By Lemma 3.5, we see that $\left.f\right|_{\mathbf{B}\left(x, d_{D}(x)\right)}$ is $M_{0}$-QH, where some $M_{0}=M_{0}(L, \lambda, \eta)$. Thus Theorem 1.2 implies that $f: D \rightarrow D^{\prime}$ is $M-\mathrm{QH}$, and hence, the proof of Theorem 1.3 is complete.

Funding. The research was partly supported by NNSFs of China (Nos. 11901090, 11571216 and 11671127) and by Department of Education of Guangdong Province, China (Grant Nos. 2018KQNCX285 and 2018KTSCX245).

\section{References}

[1] Astala, K., and F. W. Gehring: Injectivity, the BMO norm and the universal Teichmüller space. - J. Anal. Math. 46, 1986, 16-57.

[2] Beurling, A., and L. V. Ahlfors: The boundary correspondence under quasiconformal mappings. - Acta Math. 96, 1956, 125-142. 
[3] Bonk, M., J. Heinonen, and P. Koskela: Uniformizing Gromov hyperbolic spaces. Astérisque 270, 2001.

[4] Gehring, F. W.: The Carathéodory convergence theorem for quasiconformal mappings in space. - Ann. Acad. Sci. Fenn. Ser. A I Math. 336:11, 1963, 1-21.

[5] Gehring, F. W., and B. G. Osgood: Uniform domains and the quasi-hyperbolic metric. - J. Anal. Math. 36, 1979, 50-74.

[6] Gehring, F. W., and B. P. PALKa: Quasiconformally homogeneous domains. - J. Anal. Math. 30, 1976, 172-199.

[7] HuAng, X., H. LiU, and J. Liu: Local properties of quasihyperbolic mappings in metric spaces. - Ann. Acad. Sci. Fenn. Ser. A I Math. 41, 2016, 23-40.

[8] Huang, M., A. Rasila, X. Wang, and Q. Zhou: Semisolidity and locally weak quasisymmetry of homeomorphisms in metric spaces. - Stud. Math. 242, 2018, 267-301.

[9] Martio, O.: Quasisimilarities. - Rev. Roumaine Pures Appl. 36, 1991, 395-406.

[10] Rasila, A., and J. Talponen: On quasihyperbolic geodesics in Banach spaces. - Ann. Acad. Sci. Fenn. Math. 39, 2014, 163-173.

[11] Tukia, P., and J. VÄIsÄLÄ: Lipschitz and quasiconformal approximation and extension. Ann. Acad. Sci. Fenn. Ser. A I Math. 6, 1981, 303-342.

[12] VÄIsÄLÄ, J.: Free quasiconformality in Banach spaces. I. - Ann. Acad. Sci. Fenn. Ser. A I Math. 15, 1990, 355-379.

[13] VÄIsÄLÄ, J.: Free quasiconformality in Banach spaces. II. - Ann. Acad. Sci. Fenn. Ser. A I Math. 16, 1991, 255-310.

[14] VäısÄLÄ, J.: Free quasiconformality in Banach spaces. III. - Ann. Acad. Sci. Fenn. Ser. A I Math. 17, 1992, 393-408.

[15] VÄısÄLÄ, J.: Free quasiconformality in Banach spaces. IV. - Analysis and Topology, World Sci. Publ., River Edge, N. J., 1998, 697-717.

[16] VÄIsÄLÄ, J.: The free quasiworld, freely quasiconformal and related maps in Banach spaces. - In: Quasiconformal geometry and dynamics (Lublin 1996), Banach Center Publications 48, 1999, 55-118.

[17] WANG, X., and Q. ZHou: Quasimöbius maps, weakly quasimöbius maps and uniform perfectness in quasi-metric spaces. - Ann. Acad. Sci. Fenn. Math. 42, 2017, 257-284.

Received 18 August 2019• Accepted 29 April 2020 